\title{
Acupuncture versus usual care for postoperative nausea and vomiting in children after tonsillectomy/adenoidectomy: a pragmatic, multicentre, double-blinded, randomized trial
}

\author{
Ingrid Liodden $^{1}$, Leiv Sandvik ${ }^{2}$, Berit Taraldsen Valeberg ${ }^{3}$, Einar Borud ${ }^{1}$, Arne Johan Norheim ${ }^{1}$
}

${ }^{1}$ NAFKAM, Institute of Community Medicine, UiT the Arctic University of Norway, N-9037 Troms $\varnothing$,

Norway

${ }^{2}$ Faculty of odontology, University of Oslo, N-0318 Oslo, Norway

${ }^{3}$ Faculty of health service, Oslo and Akershus College University of applied science,

N-0130 Oslo, Norway

Correspondence to

Ingrid Liodden, NAFKAM, Institute of Community Medicine,

UiT the Arctic University of Norway, N-9037 Tromsø, Norway;

Ingrid@liodden.no

Key words: acupuncture, tonsillectomy children, postoperative nausea and vomiting, postoperative pain Word count: 3304

Full trial protocol can be accessed by request to corresponding author on ingrid@liodden.no 


\section{ABSTRACT}

Objectives To investigate the effect of a standardized acupuncture on nausea and vomiting in children after tonsillectomi with or without adenoidectomy when possible placebo effects were precluded. Methods A pragmatic, multicentre, double-blinded, randomized, controlled trial. The study was conducted over a 10 months in 2012-2013 at three ambulatory clinics. Two hundred and eighty-two children, age 1 to 11 years, ASA grade $\leq$ II, were included. To level out expectancy effects, all parents were told that their child would receive acupuncture. However, children were randomly allocated to peroperative bilateral needling acupuncture at PC 6, depth $7 \mathrm{~mm}$, mean time $17 \mathrm{~min}$ (SD 5 - 45) during anaesthesia plus usual care, or to usual care only. Regional ethics committee approved this approach. Primary endpoints: nausea and vomiting 24 hours postoperatively.

Results This study did not demonstrate any effect of acupuncture (95\% confidence interval) compared with standard care. The overall percentage of vomiting in the acupuncture and usual care groups were 44.2 and 47.9, respectively. Nausea was experienced by $31.7 \%$ in the acupuncture group and by 32.6 in the usual care group. The test power was acceptable regarding comparisons of vomiting.

Conclusions The findings suggest that, when controlling for possible placebo effects, a standardized PC6 acupuncture needling during anaesthesia without further stimulation of PC6 is not effective in reducing nausea and vomiting in children after tonsillectomy with or without adenoidectomy. Future studies should investigate acupuncture treatment balancing adequate dose and technique, and a feasible, child-friendly acupuncture treatment.

Trial registration ClinicalTrials.gov NCT01729052 


\section{INTRODUCTION}

Postoperative nausea and vomiting are common complications in children after tonsillectomy and adenoidectomy and remain challenges in our daily practice. Antiemetic drug treatment is not completely effective and can cause unwanted adverse events. A non-pharmacologic treatment, such as acupuncture, may therefore be of interest.

Trials on effect of acupuncture have identified conflicting results, and the quality of the methods has been debated. There is also a wide variability in acupuncture applications, such as different point of time, techniques, duration, and lack of appropriate placebo controls, making it difficult to evaluate the clinical importance. ${ }^{12}$ Metaanalyses indicate that acupuncture may reduce postoperative nausea and vomiting in adults and children. ${ }^{3-5}$

Opponents to acupuncture contend that acupuncture is not effective beyond placebo. ${ }^{67}$ The placebo effect may be defined as "a beneficial effect in a patient following a particular treatment that arises from the patient's expectations concerning the treatment rather than from the treatment itself" ${ }^{8}$ Colagiuri et.al. ${ }^{9}$ describe two forms of expectancy. The expected efficacy is to which degree a treatment is expected to work. The perceived treatment is related to randomized controlled trials constituting patients' beliefs about their group allocation. The perceived treatment is a possible source to activating or deactivating expectancies regarding treatment effect. A systematic review showed relationship between patients' expectancies and acupuncture treatment outcomes. ${ }^{10}$

The use of sham acupuncture is controversial in the research-communities. It is inclined to have high placebo effects on subjective symptoms ${ }^{11}$ and may not even be inert, as it evokes peripheral and central neural effects. ${ }^{12}$

The present study was designed to preclude possible placebo effects. First, we assumed that there was a relationship between parental treatment expectancies and treatment outcomes. Second, we assumed that parental beliefs about group allocation could activate or deactivate treatment expectancies. To remedy these biases susceptible to produce placebo effects, we told all parents that their child would receive acupuncture, while in fact they were allocated randomly to two groups. We have explored the ethical issues of this procedure in the "Ethical considerations" section. Finally, in order to make sham superfluous and thus eliminate effects of sham, we performed acupuncture during anaesthesia. 
To our knowledge, this study is the first investigating the effect of acupuncture in children when possible placebo effects of parental expectancies are controlled for.

\section{Objective}

To investigate the effect of a standardized acupuncture feasible in our busy everyday practice on nausea and vomiting in children after tonsillectomi with or without adenoidectomy when possible placebo effects were precluded.

\section{MATERIAL AND METHODS}

This is a pragmatic, multicentre, double-blinded, randomized, controlled trial. Possible placebo effects, i.e. effects of sham and expectancies, were precluded. We tested the feasibility of the study in a pilot. The protocol remained unchanged after the assessment of the pilot. Trial registry number:

ClinicalTrials.gov NCT01729052.

\section{Participants and settings}

We conducted the study at three ambulatory clinics in Norway, specialized in ear- nose- and throat surgery. The study was accomplished alongside normal practice. Inclusion criteria were children 1 to 11 years of age, with ASA (American Society of Anaesthetists) grade $\leq$ II, admitted for tonsillectomy with or without adenoidectomy. Exclusion criteria were parents requiring interpreter, rash or infection over the relevant acupuncture points, emesis or antiemetic treatment during the previous 24 hours, and gastrointestinal illness. Six anaesthetists performed the acupuncture. One of them was a trained acupuncturist; the others were trained by a licensed acupuncturist. Five experienced surgeons conducted the surgeries.

\section{Sample size}

We calculated the sample size by a program at the web pages of University of California, Department of Epidemiology and Biostatistics, San Francisco (http://www.epibiostat.ucsf.edu/biostat/sampsize.html?iframe=true \&width=100\%25\&height=100\%25\# G*Power). We based the calculation of sample size on the variable vomiting (yes/no). We expected the prevalence of vomiting to be about $50 \%$. When comparing vomiting in the two study groups, a chisquare test with $5 \%$ significance level was used. It may then be shown that in order to have $80 \%$ test power, if the true difference in prevalence of vomiting between the groups is at least 20 percentage points, ${ }^{13}$ at least 198 patients must be included in the study. We expected a dropout rate of about $30 \%$ of the included patients and decided to include 292 patients in the study. 


\section{Pre-study procedures}

The parents received study information in a letter sent beforehand, and at the day of surgery the principal researcher informed verbally. The principal researcher then enrolled the children in the study if they were eligible according to inclusion/exclusion criteria, and the parents gave written, informed consent. However, if a child would resist participation by word or action, we would respect that. The children were fasting on the day of surgery, but allowed to drink clear fluids up to two hours before surgery.

\section{Randomization}

We used a computer-generated randomization with variable block sizes of between two and six. Within each block, a 1:1 ratio allocated children to receive acupuncture plus usual care or usual care only. The anaesthetist executed the randomization by drawing the envelopes with consecutive numbers. Each new child enrolled in the study received the next consecutive envelope, which was opened after induction of anaesthesia. The information about the group assignment was not accessible to the researchers before the data collection was completed. The Clinical Research Centre, University Hospital of North Norway, prepared the randomization list.

\section{Anaesthetic management}

The anaesthetists used different modes in the administration of anaesthetic agents: 1) induction and maintenance with sevoflurane, 2) induction with sevoflurane and maintenance with propofol and remifentanyl, 3) induction and maintenance with propfol and remifentanyl. The anaesthesia was inducted and maintained with oxygen $30 \%$ and air or nitrous oxide and positive pressure ventilation. A laryngeal mask or uncuffed orotracheal tube secured the airways. All children received Ringer's acetate (at a non-standardized rate), paracetamol (suppository $40 \mathrm{mg} / \mathrm{kg}$ or intravenously $15 \mathrm{mg} / \mathrm{kg}$ ), and dexamethasone intravenously $(0,25 \mathrm{mg} / \mathrm{kg})$ or diclofenac suppository $(25 \mathrm{mg})$. The surgeons used dissection suction or diathermy scissors for tonsillectomy.

\section{Intervention and blinding}

We used acupuncture point PC6, the most frequently studied for reduction of nausea and vomiting. This may be due not to its antiemetic properties, but also to the easy access on the forearm. The anaesthetists needled, with an angle of approximately 30 degrees, the acupuncture point Neiguan (PC6) bilaterally with Seirin needles no $3(0.20) \times 15 \mathrm{~mm}$ to a depth of approximately $7 \mathrm{~mm}$ after induction of anaesthesia and removed them before entry to the recovery unit. PC6 is located at the wrist between the tendons of the palmaris longus and flexor carpi radialis, proximal from the palmar crease. There was no 
manipulation of the acupuncture needles. The needle retention time was set to 15 to 20 minutes, depending on the surgery time.

The surgery drape covered the needles (if any) peroperatively. No matter whether the child had received acupuncture or not, the anaesthetists fixed adhesive tape on the acupuncture points after needle removal. Thus, we blinded children, parents, and personnel in touch with parents and children postoperatively, such as surgeons, care providers and investigator/outcome assessor.

\section{Postoperative care}

Postoperative pain in the recovery unit was treated with fentanyl intravenously. The use of antiemetics was restricted to persistent nausea and vomiting. Ringer's acetate was continued. Two of the clinics allowed oral intake at two hours postoperatively, and one of the clinics allowed clear fluids on demand. The stay in recovery was for a minimum of one hour.

\section{Data collection}

We collected perioperative information from the anaesthesia and postoperative record. The principal investigator briefed the parents in the assessment of data. The parents assessed retching and vomiting by frequency using a purpose designed form. Recurrence of retching and vomiting within a period of two minutes was considered as one occurrence. The parents employed a behaviour tool, FLACC-N, to measure pain in children $<5$ years of age, and they assessed pain and nausea in children $>5$ years of age by using The Faces Pain Scale and the BARF nausea scale. Of interest were the highest scores during 24 hour postoperatively. They also reported their evaluation of the children's experience of overall malaise as none, minimal, moderate, great, and severe. The principal researcher collected the recorded data by telephone.

\section{Validity and reliability}

The Norwegian version of the pain assessment tool FLACC-N had a high inter-rater reliability $(0,95)$, and internal consistency reliability (Cronbach's alfa 0,96). The correlations between FLACC-N and Numeric Rating Scale indicated a moderate consistency (0.52). ${ }^{14}$ The Faces Pain Scale had a strong positive correlation $(r=0.93)$ with a visual analogue scale. ${ }^{15}$ Likewise, the Baxter Retching Faces had a strong correlation $($ Spearman $=\rho 0.93)$ with the Visual Analogue Scale for Nausea. ${ }^{16}$ 


\section{Outcome measures}

Main outcome measures were nausea and vomiting during 24 hours postoperatively. Secondary outcome measures were pain, the use of postoperative analgesics, and children's overall experience of malaise.

\section{Ethical considerations}

Written informed consent was obtained from all parents. The trial was performed in accordance with the Helsinki declaration.

All parents were told that their child would receive acupuncture, while in fact they were randomized to acupuncture or no-acupuncture. This is a major ethical consideration, and we had several discussions with the regional ethics committee (REK-nord) regarding this procedure before they recommended the study. Deception is typically used in psychology, and several official bodies have made statements on this issue. Our considerations were in accordance with National Patient Safety Agency ${ }^{17}$ and California State University ${ }^{18}$ stating that deception is justified when 1) it is not possible to obtain useful knowledge without withholding some aspects of the study protocol, 2) the findings may produce knowledge of scientific value, 3 ) use of deception must not expose the participants to more that minimal psychological or physiological risks, 4) the participants' willingness to consent is not affected. We informed the parents in the study information letter sent beforehand that some features in the protocol would not be revealed until the study was concluded.

Children may be afraid of the acupuncture needles; consequently, we performed the acupuncture during anaesthesia.

\section{Data analysis}

Retching and vomiting were collapsed into a single variable: vomiting. The continuous variables nausea and pain were converted to dichotomous variables: yes/no. When comparing percentages, the chi-square test was used. A significance level of 5\% was applied throughout. Frequencies are presented as percentage with $95 \%$ confidence interval. The data were analysed by SPSS version 21.0.

\section{RESULTS}

From November 2012 to June 2013, we included 138 children in the acupuncture group and 144 children in the control group. The consort flow diagram of the study is displayed in Figure 1. 
Figure 1 The Consort flow diagram of the study

\section{Enrollment}

$$
\text { Assessed for eligibility }(n=340)
$$
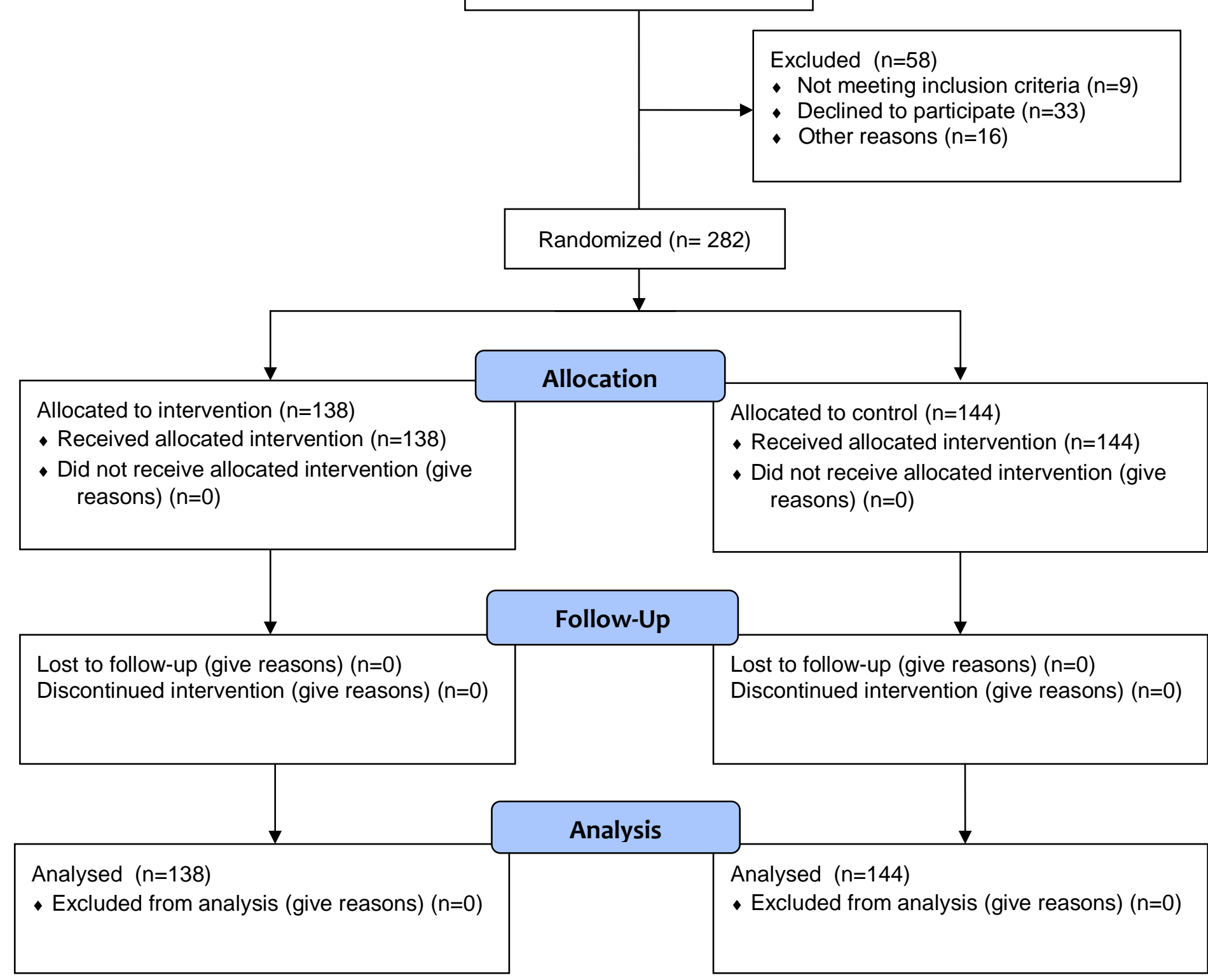

Patients' characteristics, type and duration of surgery/needle retention time are presented in table 1.

Types of anaesthesia, use of laryngeal mask or orotracheal tube, surgery techniques, and administration of intravenous fluids were evenly distributed in the two groups. One child was readmitted to hospital because of bleeding. 
Table 1 Baseline characteristics and peroperative data. Values are numbers (\%) unless stated otherwise

\begin{tabular}{|c|c|c|c|c|}
\hline \multicolumn{3}{|c|}{ Characteristic } & $\begin{array}{l}\text { Acupuncture } \\
(\mathrm{n}=138)\end{array}$ & $\begin{array}{l}\text { Usual care } \\
(\mathrm{n}=144)\end{array}$ \\
\hline \multirow[t]{2}{*}{ Gender } & \multicolumn{2}{|c|}{ Boys } & $52 / 119(37.7)$ & $67 / 119(46.5)$ \\
\hline & \multicolumn{2}{|c|}{ Girls } & $86 / 163(62.3)$ & $77 / 163(53.5)$ \\
\hline \multicolumn{3}{|c|}{ Age (year) mean (range) } & $4.3(1-11)$ & $4.5(1-11)$ \\
\hline \multicolumn{3}{|c|}{ History of motion sickness } & 42/83 (30.4) & $41 / 83(28.5)$ \\
\hline \multicolumn{3}{|c|}{$\begin{array}{l}\text { History of postoperative } \\
\text { nausea and vomiting }\end{array}$} & 5/17 (10.4) & $12 / 17(24.0)$ \\
\hline \multirow{2}{*}{\multicolumn{2}{|c|}{ Type of surgery }} & Adenoidectomy & $73 / 148(52.9)$ & $75 / 148(52.1)$ \\
\hline & & $\begin{array}{l}\text { Tonsillectomy/ } \\
\text { adenotonsillectomy }\end{array}$ & 65/134 (48.5) & $69 / 134(51.5)$ \\
\hline \multicolumn{3}{|c|}{$\begin{array}{l}\text { Duration of surgery }(\min ) \approx \text { needle } \\
\text { retention time mean }(\text { range })\end{array}$} & $17(5-45)$ & $18(3-45)$ \\
\hline
\end{tabular}

This study did not demonstrate any significant differences in overall vomiting ( 0 to 24 hours) or in early ( 0 to 6 hours) and late ( 6 to 24 hours) between the acupuncture and usual care group. Likewise, the study showed no effect of acupuncture on nausea (in children $>5$ years). The overall percentages of vomiting in the acupuncture and usual care groups were 44.2 and 47.9, respectively. Nausea was experienced by $31.7 \%$ in the acupuncture group and by 32.6 in the usual care group (Table 2). Subgroup analysis showed no significant association between gender and vomiting ( acupuncture group $\mathrm{p}=0.441$, usual care group $\mathrm{p}=0.166$ ) or gender and nausea (acupuncture group $\mathrm{p}=0.658$, usual care group $\mathrm{p}=$ 0.901). The lengths of the $95 \%$ confidence interval for the frequency differences were 23.2 for overall vomiting and 39.4 for nausea. 
Table 2 Primary outcomes: vomiting and nausea. Values are numbers (\%) unless stated otherwise

\begin{tabular}{|c|c|c|c|c|}
\hline Outcomes & Acupuncture & Usual care & $\begin{array}{l}\text { Frequency } \\
\text { difference }\end{array}$ & P-value \\
\hline Overall vomiting (24 hours) & $61 / 138(44.2)$ & $69 / 144(47.9)$ & 3.7 & 0.532 \\
\hline $95 \%$ confidence interval & 35.5 to 52.9 & 39.6 to 55.6 & -15.3 to 7.9 & \\
\hline Early vomiting ( 0 to 6 hours) & $41 / 138(29.7)$ & $50 / 144(34.7)$ & 5.0 & 0.347 \\
\hline $95 \%$ confidence interval & 21.7 to 37.7 & 27.1 to 42.2 & -15.9 to 5.9 & \\
\hline Late vomiting ( 7 to 24 hours) & $37 / 138(26.8)$ & $40 / 144(27.8)$ & 1.0 & 0.856 \\
\hline $95 \%$ confidence interval' & 18.8 to 34.8 & 20.1 to 34.7 & -11.4 to 9.4 & \\
\hline Nausea, children $>5$ years & 13/41 (31.7) & $15 / 46(32.6)$ & 0.9 & 0.928 \\
\hline $95 \%$ confidence interval & 19.5 to 46.3 & 21.7 to 45.7 & - 20.6 to 18.8 & \\
\hline
\end{tabular}

*Two different pain assessment tools: The Faces Pain Scale and FLACC, both with a scale from 0 to 10

The lack of differences in the two groups was present also in the cases when the anaesthetist who was a trained acupuncturist, performed acupuncture (p-values for vomiting and nausea: 0.679 and 0.348 respectively). There was no difference in the two groups concerning children's experience of pain and malaise, or the use of analgesics postoperatively (Table 3).

The parents reported no discomfort or dermatological problems at the acupuncture site. However, they observed minor hematomas in 15 children. 
Table 3 Secondary outcomes: Pain, use of analgesics postoperatively and children's overall malaise. Values are numbers (\%) unless stated otherwise

\begin{tabular}{|c|c|c|c|c|}
\hline Variable & $\begin{array}{c}\text { Acupuncture } \\
\mathrm{n}=138\end{array}$ & $\begin{array}{c}\text { Usual care } \\
\mathrm{n}=144\end{array}$ & $\begin{array}{l}\text { Frequency } \\
\text { difference }\end{array}$ & P-value \\
\hline Pain & $107 / 138(77.5)$ & $104 / 144(72.2)$ & 5.3 & 0.304 \\
\hline $95 \%$ confidence interval & 70.3 to 84.4 & 65.3 to 79.2 & -4.8 to 15.4 & \\
\hline Fentanyl at recovery unit & $59(42.8 \%)$ & $65(45.1 \%)$ & 2.39 & 0.687 \\
\hline $95 \%$ confidence interval & & & - 14.0 to 9.2 & \\
\hline Analgesics* post discharge & $102(73.9 \%)$ & $98(68.1 \%)$ & 5.6 & 0,279 \\
\hline $95 \%$ confidence interval & & & - 4.7 to 16.4 & \\
\hline Minimal to moderate & $96(69.6 \%)$ & $90(62.5 \%)$ & 7.1 & 0,432 \\
\hline $95 \%$ confidence interval & & & -4.0 to 18.1 & \\
\hline Great to severe & $18(13.0 \%)$ & $25(17.4 \%)$ & 4.3 & \\
\hline $95 \%$ confidence interval & & & - 12.7 to 4.0 & \\
\hline
\end{tabular}

*Paracetamol, codeine phosphate, NSAIDS - single medication or in combination

\section{DISCUSSION}

The present study did not demonstrate any significant difference between needling acupuncture point PC 6 during anaesthesia plus usual care versus usual care only, as regards to postoperative vomiting and nausea after tonsillectomy with or without adenoidectomy in children. The length of the confidence interval for difference in vomiting between the groups were relatively small, showing that the test power of the study was acceptable as regards comparisons of vomiting. ${ }^{19}$ There was no difference in the two groups concerning children's experience of pain and malaise, or the use of analgesics postoperatively.

It has been argued that the possibility of obtaining significant results when comparing two groups is increased when the efficacy variables are measured on a continuous or discrete scale, as compared to a dichotomized scale. The effect variables measuring vomiting, nausea and pain were originally measured on discrete and continuous scales. We have compared and analysed these original variables using Mann-Whitney U-test, and the results from these analyses were far from significant.

The lack of evidence of antiemetic effect in this trial complies with a similar double-blind trial by Yentis $^{20}$ who concluded that P6 acupuncture during anaesthesia is ineffective compared to nonacupuncture in reducing vomiting after tonsillectomy in children. Shenkman ${ }^{21}$ considered that 
acupuncture might require an intact nervous system, which again requires an awake state of the patient. Accordingly, he conducted a double-blind trial including acupuncture at PC6 during anaesthesia and acupressure preoperatively compared with sham in children scheduled for tonsillectomy.

Notwithstanding, the combination of acupressure and acupuncture peroperatively compared to sham was ineffective for emesis.

To the contrary, Somri et al. ${ }^{22}$ concluded in their double-blinded trial that there was a significant difference in PONV after dental surgery in children who received acupuncture compared to sham acupuncture during anaesthesia. However, Somri used PC6 and CV13. A combined use seems to be more effective compared with single use. ${ }^{23}$ Another trial on children scheduled for tonsillectomy and adenoidectomy has demonstrated the effect of acupuncture during anaesthesia and acupressure postoperatively. ${ }^{13}$ However, in this trial, the control was not sham but usual care, and the parents and investigators were not blinded.

Gan et al. ${ }^{24}$ found that transcutaneous electrostimulation at PC6 before and during surgery, in addition to preventing PONV, also appeared to provide analgesia in adults. We have found little support suggesting that the present acupuncture modality is less effective compared to other modalities. Lee et al. ${ }^{4}$ have suggested in their systematic review that invasive (e.g. acupuncture, electrical stimulation) and non-invasive (e.g. acupressure, transcutaneous electrical stimulation) had similar effects.

Studies comparing acupuncture with sham often fail to demonstrate any differences of effect. It has been suggested that effects following both real and sham acupuncture result from a variety of active components, and that sham actually acts as an active treatment. ${ }^{11}$ We avoided this bias by using usual care as control, yet we found no difference between the two groups.

The strength of this study is that the methods allowed us to preclude possible placebo effects. Other strengths are the large sample size, the large follow-up rate, and a successful blinding; only one parent called in question whether the child had received acupuncture.

There are some limitations in this study, reducing internal validity. The variability of anaesthetic management and other medications may lead to different results; volatiles and opioids may contribute to, whereas propofol may reduce, emesis. Further, laryngeal mask and orotracheal tube produce different reactions in terms of experience of malaise and vomiting. The different postoperative care strategies concerning oral intake of fluids may also affect the outcomes. Nevertheless, one of the intentions in a pragmatic trial is to try to answer the question Can this intervention be useful in our clinical practice? One strategy in answering this is to secure a strong external validity by including 
patients from as many sources as possible (e.g. clinics) and attain flexibility in terms of intervention and comparison. ${ }^{25} \mathrm{We}$ might have taken these factors into consideration when estimating the sample size; a larger study would allow for greater variation between centres. The sample size calculation was based on a difference of 20 percentage points based on results on a previous study. ${ }^{13}$ In the present study, however, we might expect a smaller effect as the expectancy effect was controlled for. Recognizing these considerations, the study may be underpowered.

The acupuncture procedure was standardized. The only stimulation was at insertion of the needles during anaesthesia. Performing acupuncture during anaesthesia precluded the needle sensation (de qi) and might cause problems in achieving the accuracy of identifying the acupoint PC. One may speculate whether this could have produced negligible neuro-physiological effect and thus minimal likelihood of benefit. The evidence of acupuncture point specificity is conflicting. A review on studies on acupuncture points and sham points or non-specific acupuncture points confirmed the existence of acupuncture point specificity. ${ }^{26}$ In contrast, a systematic review of the randomized controlled trials with sham acupuncture controls did not find any acupuncture point specificity. ${ }^{27}$ Moreover, Somri ${ }^{22}$ found a statistically significant antiemetic effect of a standardized acupuncture at two acupuncture points without stimulation during anaesthesia. In the present study, the duration of the acupuncture treatment varied according to surgery time. Nevertheless, relatively short needle retention times $(1,5$, and 15 minutes, respectively) have been demonstrated effective in children. 2228

Female gender is considered a risk factor for PONV in adults, ${ }^{30}$ but not in children. ${ }^{31}$ However, a previous study found that girls were more prone to vomiting than were boys. ${ }^{13}$ In the present study, there is an imbalance of girls/boys in the two groups, as a preponderance of girls received acupuncture. There was no significant association between gender and nausea or vomiting, so gender was not a confounding factor.

Thirty-three out of 340 parents declined to participate, and nine parents were in need of an interpreter. These numbers are small, suggesting negligible selection bias. Patients often wish to make things turn out well, a notion of "eager-to-please", which may cause information bias. This bias was ruled out in the two groups, as all parents believed that their child received acupuncture treatment. The telephone interview is a possible source of bias, as the principal researcher might subconsciously influence the parents' responses. In order to minimize this flaw, we used a structured interview.

The aim of this pragmatic trial was to investigate the effect of acupuncture treatment feasible in our busy everyday practice. We consider that feasibility involves a standardized acupuncture procedure that do not demand any extra time and recourses, and is easy to learn and perform by the existing 
department personnel. Last, but not least, it must be acceptable for the children, and needling while the child was awake was thus not an option. The acupuncture dose in this study may be considered inadequate, biasing the results. Feasibility and child-friendliness should not equate to an inadequate acupuncture. To remedy this and optimize a possible acustimulation effect, we have previously performed an open pragmatic study designed by using acupuncture during anaesthesia and wristbands postoperatively, ${ }^{13}$ and found that vomiting was significantly less pronounced in children receiving acupuncture relative to children receiving usual care. One may speculate whether these results were due to the additional use of wristbands, or to the open design allowing the influence of parental expectancy be part of the effect, or due to both. We suggest future studies should investigate acupuncture treatment balancing adequate dose and technique, and a feasible, child-friendly acupuncture treatment. Usage of several acustimulation points, stimulation of needles, and additional acupressure wrist-bands are characteristics that may increase acupuncture dose and may be exciting areas for future investigation.

\section{Conclusion}

The findings suggest that, when controlling for possible placebo effects, a standardized PC6 acupuncture needling during anaesthesia without further stimulation is not effective in reducing nausea and vomiting in children after tonsillectomy with or without adenoidectomy. Future studies investigate acupuncture treatment balancing adequate dose and technique, and a feasible, child-friendly acupuncture treatment.

\section{Summary points}

- Acupuncture needling at PC6 was compared with standard care for postoperative nausea and vomiting in children after tonsillectomy with or without adenoidectomy.

- Standardized PC6 acupuncture needling during anaesthesia without stimulation was not effective when controlling for possible placebo effects.Future studies should investigate acupuncture treatment balancing adequate dose and technique, and a feasible, child-friendly acupuncture treatment.

\section{Contributors}

IL had the main responsibility for the conception and design of the work and the acquisition; she contributed to the data analysis and interpretation, and wrote the paper. LS had the main responsibility for the data analyses and interpretation, and contributed to the writing. BTV contributed to the design and writing the paper. EB contributed to the design and data analysis. AJN contributed to the 
conception and design, analysis, interpretation, and the writing. All contributors have revised the work, approved the final version and agreed to be accountable for all aspects of the work.

Acknowledgements The authors thank the staff at the outpatient surgery departments, and the anaesthetists for performing the acupuncture. A special thanks to the children and their parents who participated in the study. We collected the data at Nydalen Øre- Nese- Hals, Asker Øre- NeseHalsklinikk AS, and Polikliniske Operasjoner AS.

Competing interest None declared.

Patient consent Obtained.

Ethics approval Regional Ethics Committee (2012/63/REK nord).

Provenance and peer review Not commissioned; externally peer reviewed.

\section{REFERENCES}

1. Chernyak GV, Sessler DI. Perioperative acupuncture and related techniques. Anesthesiology 2005;102(5):1031-49.

2. Streitberger K, Kranke P. Evidence for the efficacy of acupressure for preventing post-operative nausea and vomiting: an ongoing debate. European journal of anaesthesiology 2011;28(6):3968.

3. Ezzo J, Streitberger K, Schneider A. Cochrane systematic reviews examine P6 acupuncture-point stimulation for nausea and vomiting. Journal of alternative and complementary medicine 2006;12(5):489-95.

4. Lee A, Fan LT. Stimulation of the wrist acupuncture point P6 for preventing postoperative nausea and vomiting. Cochrane Database Syst Rev 2009(2):1-86.

5. Dune LS, Shiao SY. Metaanalysis of acustimulation effects on postoperative nausea and vomiting in children. Explore (NY) 2006;2(4):314-20.

6. Pandolfi M. The autumn of acupuncture. European journal of internal medicine 2012;23(1):31-3.

7. O'Connell NE, Wand BM, Goldacre B. Interpretive bias in acupuncture research?: A case study. Evaluation \& the health professions 2009;32(4):393-409.

8. American Heritage. Stedman's Medical Dictionary. Secondary Stedman's Medical Dictionary 2009. http://dictionary.reference.com/browse/placebo

9. Colagiuri B. Participant expectancies in double-blind randomized placebo-controlled trials: potential limitations to trial validity. Clin Trials 2010;7(3):246-55.

10. Colagiuri B, Smith CA. A systematic review of the effect of expectancy on treatment responses to acupuncture. Evidence-based complementary and alternative medicine 2012;2012:857804.

11. Lundeberg T, Lund I, Naslund J, et al. The Emperor's sham - wrong assumption that sham needling is sham. Acupunct Med 2008;26(4):239-42.

12. Rycroft-Malone J, Seers K, Titchen A, et al. What counts as evidence in evidence-based practice? Journal of advanced nursing 2004;47(1):81-90. 
13. Liodden I, Howley M, Grimsgaard AS, et al. Perioperative acupuncture and postoperative acupressure can prevent postoperative vomiting following paediatric tonsillectomy or adenoidectomy: a pragmatic randomised controlled trial. Acupuncture in medicine : journal of the British Medical Acupuncture Society 2011;29(1):9-15.

14. Reinertsen H, Christophersen K, Helseth S. Validering og reliabilitetstesting av smertevurderingsverktøyet FLACC. Sykepeleien Forskning 2014;9(2):132-40.

15. Hicks CL, von Baeyer CL, Spafford PA, et al. The Faces Pain Scale-Revised: toward a common metric in pediatric pain measurement. Pain 2001;93(2):173-83.

16. Baxter AL, Watcha MF, Baxter WV, et al. Development and validation of a pictorial nausea rating scale for children. Pediatrics 2011;127(6):1542-9.

17. National-Patient-Safety-Agency. Issues, giudance and evidence - deception in medical research. Secondary Issues, giudance and evidence - deception in medical research 2009. http://www.hra.nhs.uk/documents/2013/09/ige-paper-deception-in-medical-research.pdf.

18. California-state-University. Use of deception in research. Secondary Use of deception in research 2014. http://www20.csueastbay.edu/orsp/forms-policies-procedures/irb/deception.html.

19. Machin D CM, Tan S-B. Sample Size Tables for Clinical Studies. 3 ed. Sussex, UK: John WileyBlackwell, 2009.

20. Yentis SM, Bissonnette B. P6 acupuncture and postoperative vomiting after tonsillectomy in children. British journal of anaesthesia 1991;67(6):779-80.

21. Shenkman Z, Holzman RS, Kim C, et al. Acupressure-acupuncture antiemetic prophylaxis in children undergoing tonsillectomy. Anesthesiology 1999;90(5):1311-6.

22. Somri M, Vaida SJ, Sabo E, et al. Acupuncture versus ondansetron in the prevention of postoperative vomiting. A study of children undergoing dental surgery. Anaesthesia 2001;56(10):927-32.

23. Alizadeh R, Esmaeili S, Shoar S, et al. Acupuncture in Preventing Postoperative Nausea and Vomiting: Efficacy of Two Acupuncture Points Versus a Single One. J Acupunct Meridian Stud 2013(0).

24. Gan TJ, Jiao KR, Zenn M, et al. A randomized controlled comparison of electro-acupoint stimulation or ondansetron versus placebo for the prevention of postoperative nausea and vomiting. Anesthesia and analgesia 2004;99(4):1070-5.

25. Thorpe KE, Zwarenstein M, Oxman AD, et al. A pragmatic-explanatory continuum indicator summary (PRECIS): a tool to help trial designers. Cmaj 2009;180(10):E47-57.

26. Rong PJ, Zhao JJ, Gao JH, et al. Progress of research on specificity of meridian acupoint efficacy. Chinese journal of integrative medicine 2013;19(12):889-93.

27. Zhang H, Bian Z, Lin Z. Are acupoints specific for diseases? A systematic review of the randomized controlled trials with sham acupuncture controls. Chinese medicine 2010;5:1.

28. Butkovic D, Toljan S, Matolic M, et al. Comparison of laser acupuncture and metoclopramide in PONV prevention in children. Paediatric anaesthesia 2005;15(1):37-40.

29. Kabalak AA, Akcay M, Akcay F, et al. Transcutaneous electrical acupoint stimulation versus ondansetron in the prevention of postoperative vomiting following pediatric tonsillectomy. $\mathrm{J}$ Altern Complement Med 2005;11(3):407-13.

30. Apfel CC, Heidrich FM, Jukar-Rao S, et al. Evidence-based analysis of risk factors for postoperative nausea and vomiting. British journal of anaesthesia 2012;109(5):742-53.

31. Eberhart LH, Geldner G, Kranke P, et al. The development and validation of a risk score to predict the probability of postoperative vomiting in pediatric patients. Anesth Analg 2004;99(6):1630-7. 